\title{
Riqueza e fitossociologia de plantas vasculares em dunas costeiras de Torres, Rio Grande do Sul, Brasil
}

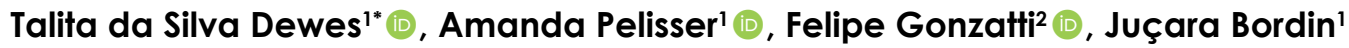 \\ ' Universidade Estadual do Rio Grande do Sul - Unidade Hortênsias, Rua Assis Brasil, 842, \\ Centro, 95400-000, São Francisco de Paula, Rio Grande do Sul, Brasil. \\ ${ }^{2}$ Universidade de Caxias do Sul - Herbário da Universidade de Caxias do Sul, Rua Francisco Getúlio Vargas, \\ 1130, Caxias do Sul, Rio Grande do Sul, Brasil. \\ *Autor para correspondência: talita-dewes@vergs.edu.br
}

Recebido em 15.VII.2019

Aceito em 26.X.2020

DOI 10.21826/2446-82312021v76e2021001

\begin{abstract}
RESUMO - Os aspectos geomorfológicos e climáticos influem no mosaico de ambientes existentes na região norte do Rio Grande do Sul, do qual destacam-se as dunas costeiras. O objetivo deste estudo foi realizar o levantamento florístico, através de caminhamento, e caracterizar a estrutura vegetacional por meio da avaliação de parâmetros fitossociológicos em 45 parcelas, de uma porção de dunas costeiras, Torres, RS. Foram identificadas 141 espécies, 104 nativas e 37 exóticas, distribuídas em 54 famílias. As famílias Asteraceae e Poaceae apresentaram a maior riqueza florística. As espécies que apresentaram maior valor de importância foram Panicum racemosum (P. Beauv.) Spreng. e Panicum repens L. O alto número de espécies encontradas bem como a presença de espécies ameaçadas de extinção indica a importância da área e a necessidade de ampliação dos esforços para a conservação, principalmente por tratar-se de uma zona de amortecimento da unidade de conservação Refúgio da Vida Silvestre Ilha dos Lobos.
\end{abstract}

Palavras-chave: florística, restinga, vegetação exótica.

\begin{abstract}
Richness and phytosociology of vascular plants in coastal dunes of Torres, Rio Grande do Sul, Brazil. The geomorphological and climatic aspects influence the environments mosaic existing in the northern region of Rio Grande do Sul, of which the coastal dunes stand out. The aim of this study was to carry out the floristic survey, by means of walks, and characterize the vegetation structure through the evaluation of phytosociological parameters in 45 plots, in a portion of coastal dunes, Torres, RS. A number of 141 species were identified, 104 native and 37 exotics, distributed in 54 families. The families Asteraceae and Poaceae presented the highest floristic richness. The species with the highest value of importance were Panicum racemosum (P. Beauv.) Spreng. and Panicum repens L. The high number of species found as well as the presence of endangered species indicates the importance of the area and the need to expand efforts for conservation, mainly because it is a buffer zone of the conservation unit Ilha dos Lobos Wild Life Refuge.
\end{abstract}

Keywords: exotic vegetation, floristic survey, restinga.

\section{INTRODUÇÃO}

Ao longo dos ecossistemas costeiros e marinhos da América, África, Ásia e Oceania estima-se que ocorram cerca 1.900 espécies animais e vegetais (Martinéz et al. 2007). Estes ecossistemas, e sua biodiversidade associada, representam mais de $60 \%$ do valor econômico da biosfera, por prestar uma série de serviços ecológicos (Liquete et al. 2013), que incluem desde servir como berçário e habitat para inúmeras espécies, manter a estabilidade do solo, servir como filtro para as águas continentais, fixação do carbono atmosférico, até como barreira e atenuante na dinâmica das marés (Worm et al. 2006, Barbier et al. 2011, Duarte et al. 2013). Somados, estes serviços ecológicos podem corresponder a cerca de dois milhões de dólares por $\mathrm{km}^{2} /$ ano em ambientes naturais (Martinéz et al. 2007).

A costa brasileira estende-se por $7.367 \mathrm{~km}$ ao longo do Oceano Atlântico, desde o Oiapoque até o Chuí (Lacerda et al. 1993). Nela pode-se observar uma grande variação de ambientes, que variam de acordo com a matriz geológica que os compõem, podendo apresentar desde costões rochosos até extensos bancos de dunas (Rizzini 1997). Neste contexto, a região costeira do Rio Grande do Sul diferencia-se das demais do Brasil, uma vez que, a partir do Cabo de Santa Marta (Laguna, Santa Catarina) até a região de La Coronilla (Rocha, Uruguai) (Seeliger 1992), a costa apresenta-se como uma ampla planície de origem Quaternária formada através dos eventos de transgressão e regressão marinhos ocorridos no Holoceno (Tomazelli et al. 2000, Villwock \& Tomazelli 2007). Além dos extensos bancos de dunas deposicionais, no extremo norte do litoral do Rio Grande do Sul, na região de Torres, encontramse exposições de rochas ígneas metamórficas provindas do derramamento basáltico da Serra Geral (Planalto das Araucárias), que dão origem a costões rochosos junto à linha da costa (Buchmann et al. 2009). 
Os ambientes costeiros têm fornecido ao longo do tempo uma série de usos às populações que se fixam junto a eles, principalmente os recursos associados ao extrativismo de espécies, bem como para recreação (Barbier et al. 2011). Isso tem aumentado a supressão da vegetação, a perda das espécies associadas, e os processos de desequilíbrio ambiental, principalmente nos estoques de carbono (Duarte et al. 2013). Além disso, observa-se o descontrole sobre os processos erosivos, a extinção das espécies nativas em função da invasão por espécies exóticas e a perda dos serviços ecossistêmicos associados (Worm et al. 2006, Martinéz et al. 2007).

A vegetação que ocorre nas formações litorâneas é comumente chamada de vegetação de restinga ou vegetação de formações pioneiras. Esta vegetação, especialmente no Litoral Norte do estado do Rio Grande do Sul, corresponde a uma fitofisionomia do domínio da Mata Atlântica, onde a maioria das espécies está adaptada a habitar ambientes com alta variação nas condições ecológicas de insolação, disponibilidade hídrica, estresse salino e eólico, escassez nutricional e possui histórico biogeográfico em comum (Leite \& Klein 1990, Lacerda 1993, Scarano 2002, Fiaschi \& Pirani 2009, IBGE 2019). A região de Torres, no extremo norte do litoral gaúcho, constituiu um importante corredor de conexão e migração da flora tropical do Sudeste (Floresta Ombrófila Densa) com as planícies e flancos da Serra Geral do Rio Grande do Sul (Rambo 1950), como demonstram estudos palinológicos conduzidos por Lorscheitter (2003).

Diversos aspectos fitogeográficos têm sido descritos por diferentes autores como Rambo (1951, 1954, 1956), Klein (1984), Waechter (1985, 1990, 1998, 2002), Scherer (2009), Gonzatti et al. (2016), e têm despertado interesse na realização de inventários pontuais ou envolvendo toda planície, dos mais diferentes tipos de vegetação ou sinúsias específicas. Tais iniciativas melhoram a base amostral da vegetação ocorrente nestas áreas, bem como fornecem dados para a avaliação do estado de conservação das espécies em nível local e regional. Contudo, muitas áreas ainda carecem de dados detalhados sobre a composição florística atualmente existente.

Considerando somente os ambientes de dunas costeiras frontais, os quais fazem parte das restingas do Rio Grande do Sul, inventários florísticos que descrevem sua vegetação, foram conduzidos por Valls (1975) e Lindeman et al. (1975) no Parque Estadual de Torres, e Pfadenhauer \& Ramos (1979) nas dunas de Tramandaí, Cordazzo \& Seeliger (1987) nas dunas de Rio Grande, Seeliger (1992) nas dunas frontais desde a região do Cassino (Rio Grande) até o Hermenegildo (Santa Vitória do Palmar), Duarte \& Bencke (2006) no plano de manejo do Parque Estadual do Itapeva, em Torres e Palma \& Jarenkow (2008) no Parque Estadual do Itapeva, também no município de Torres.

Visando o incremento de informações acerca da composição florística das dunas costeiras do litoral sulbrasileiro, o presente estudo teve como objetivo principal identificar a composição de espécies da flora vascular de dunas costeiras no município de Torres, norte do Rio Grande do Sul, Brasil. Dados acerca do status de conservação das espécies e da estrutura da comunidade de plantas em dunas costeiras também são apresentados e discutidos.

\section{MATERIAL E MÉTODOS}

\section{Área de estudo}

A área de estudo compreende um cordão de dunas vegetadas, composto por dunas embrionárias e dunas frontais de acordo com a classificação proposta por Tomazelli (1994), com cerca de dois quilômetros de extensão e $200 \mathrm{~m}$ de largura (Fig. 1), situado no município de Torres, litoral norte do Rio Grande do Sul, Brasil. Trata-se de uma porção da zona costeira que vai da foz do Rio Mampituba, ao norte, até o Morro da Guarita, ao sul, denominada Praia dos Molhes e Praia Grande (entre

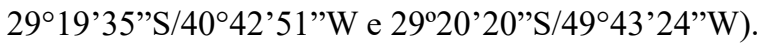

O cordão de dunas inicia próximo aos molhes do Rio Mampituba, ao norte, e encontra-se entre a faixa de praia, a leste, e um passeio público pavimentado, a oeste, que possui alguns quiosques, restaurantes e chuveiros ao longo de sua extensão, limítrofe a Avenida Beira-mar. Há canais eventuais de drenagem pluvial que segmentam o cordão de dunas e, também, passarelas construídas em madeira para acesso dos banhistas à praia.

Segundo Falcão (2000), o passeio público denominado calçadão, foi construído no final da década de 70 , assim como os molhes, obra de fixação da desembocadura do Rio Mampituba (Zasso 2012). A exploração turística, iniciada por volta de 1910, influenciou o desenvolvimento do mercado imobiliário e da construção civil no município, fazendo com que por volta da década de 60 já existissem inúmeras residências nas proximidades da orla destinadas, predominantemente, a veraneio e locação (Falcão 2000).

Com intuito de conservar a visibilidade da praia, em dezembro de 1962 foi promulgada a Lei $n^{\circ} 728 / 1962$ estabelecendo o limite de dois pavimentos e a finalidade de habitação unifamiliar para as construções próximas à orla marítima (Falcão 2000, Graciano 2004). No entanto, as alterações dos dispositivos legais referentes às normas de construção bem como a demanda dos turistas, influenciaram a expansão e construção de estabelecimentos comerciais e de hospedagem nas proximidades da orla por volta dos anos 80 (Falcão 2000, Graciano 2004). O contexto de uso desta área permanece com foco turístico ainda hoje, destacando-se as atividades de hospedagem, gastronomia e atividades recreativas como surfe e pesca.

\section{Amostragem e análise de dados}

O levantamento de dados foi realizado entre agosto de 2016 e novembro de 2017. Para a avaliação fitossociológica, foram amostradas 45 parcelas de $1 \mathrm{~m}^{2}$ com intervalos de $25 \mathrm{~m}$, distribuídas em oito transecções perpendiculares à linha da costa, ao longo da extensão da área de estudo. 

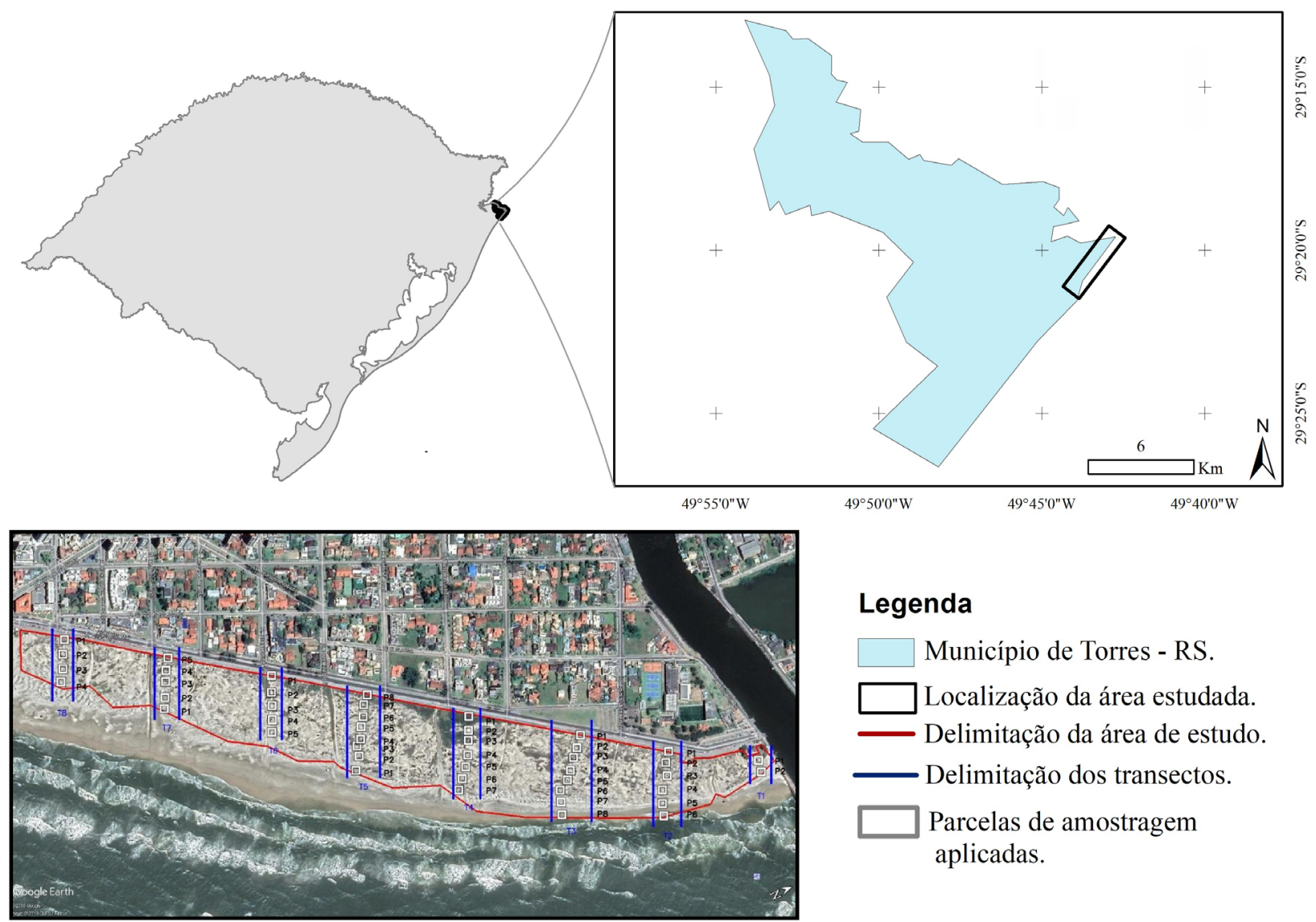

\section{Legenda}

$\square$ Município de Torres - RS.

Localização da área estudada.

Delimitação da área de estudo.

Delimitação dos transectos.

Parcelas de amostragem aplicadas.

Figura 1. Área de estudo com a localização das transecções e parcelas amostradas. Torres, Rio Grande do Sul, Brasil.

$\mathrm{Na}$ análise das unidades amostrais foram observadas as espécies da flora vascular presentes e estimou-se sua respectiva cobertura, registrada através da estimativa do percentual ocupado. Realizou-se também o caminhamento pela área de estudo (Filgueiras et al. 1994), para observação e registro das espécies não avistadas nas parcelas.

Os espécimes foram observados, fotografados e quando férteis coletados para identificação e confecção das exsicatas incorporadas ao acervo do Herbário Dr. Ronaldo Wasum da Universidade Estadual do Rio Grande do Sul (HERW). Para identificação das espécies utilizou-se chaves dicotômicas e descrições apresentadas em obras especializadas para cada grupo taxonômico, consulta na plataforma Flora do Brasil 2020 (BFG 2015), comparações com material de herbário físico ou através da plataforma INCT Herbário Virtual e REFLORA. A redação nomenclatural seguiu Brummitt \& Powell (1992) e o posicionamento taxonômico de gêneros e espécies seguiu a Flora do Brasil 2020. Também foram consultadas a origem e a distribuição geográfica das espécies no Brasil, conforme a plataforma da Flora do Brasil 2020 e obras de revisão taxonômica especializadas.

O status de conservação das espécies registradas na área de estudo foi consultado no Decreto Estadual n ${ }^{\circ}$ 52.109, de 19 de dezembro de 2014- Lista das espécies da flora nativa ameaçadas de extinção (Rio Grande do Sul 2014) e na plataforma da Flora do Brasil 2020. A classificação das espécies exóticas seguiu a Portaria SEMA n ${ }^{\circ} 79$ de 31 de outubro de 2013 (Rio Grande do Sul 2013).

Para análise fitossociológica, foram considerados os parâmetros de riqueza, frequência e cobertura absolutas e relativas, e valor de importância (Mueller-Dombois \& Ellenberg 1974, Schneider \& Irgang 2005). O valor de importância foi divido por dois pois o parâmetro densidade não foi calculado devido à dificuldade em diferenciar indivíduos da maioria das espécies herbáceas. Para as análises estatísticas foram utilizados os programas Excel e Past versão 1.73 (Hammer et al. 2001).

\section{RESULTADOS E DISCUSSÃO}

Foram registradas 141 espécies de plantas vasculares na área de estudo, sendo 104 nativas e 37 exóticas, distribuídas em 54 famílias (Tab. 1). As famílias com maior número de representantes foram Asteraceae ( $25 \mathrm{spp} ., 17,7 \%$ ), Poaceae $(15,10,6 \%)$, Asparagaceae (sete, $4,9 \%)$, Solanaceae (seis, 4,2\%), Cyperaceae e Fabaceae com cinco $(3,5 \%)$ cada, Myrtaceae e Verbenaceae com quatro espécies $(2,8 \%)$ cada. 
Tabela 1. Lista de espécies por família botânica identificadas no Levantamento Florístico das dunas costeiras de Torres, Rio Grande do Sul, Brasil. Status de conservação: EN= Em perigo, VU= Vulnerável, $\mathrm{NT}=$ Quase ameaçada. * Espécie naturalizada. ** Espécie exótica. *** Ocorrência citada por Barbosa (2017) e I3N Brasil (2019). **** Ocorrência no RS citada por Biondo et al. (2018).

\begin{tabular}{|c|c|c|c|}
\hline \multirow{2}{*}{ Família/ Espécie } & \multicolumn{2}{|c|}{ Status } & \multirow{2}{*}{ Padrão de distribuição geográfico no Brasil } \\
\hline & $\mathrm{BR}$ & $\mathrm{RS}$ & \\
\hline \multicolumn{4}{|l|}{ Achantaceae } \\
\hline Odontonema tubaeforme (Bertol.) Kuntze ** & - & - & DESCONHECIDO \\
\hline \multicolumn{4}{|l|}{ Aizoaceae } \\
\hline Carpobrotus edulis (L.) N. E. Br.* & - & - & $\mathrm{RS}, \mathrm{SC}$ \\
\hline Tetragonia tetragnoides (Pall.) Kuntze* & - & - & BA, CE, PE, MG, RJ, SP, PR, RS, SC \\
\hline \multicolumn{4}{|l|}{ Amaranthaceae } \\
\hline Alternanthera philoxeroides (Mart.) Griseb. & - & - & $\begin{array}{l}\text { AM, AP, PA, AL, BA, PB, PE, RN, SE, MS, MT, MG, ES, SP, PR, } \\
\text { RS, SC }\end{array}$ \\
\hline Blutaparon portulacoides (St.-Hil.) Mears & $\mathrm{VU}$ & - & AL, BA, CE, MA, PB, PE, RN, SE, ES, RJ, SP, PR, RS, SC \\
\hline Gomphrena perennis L. & NT & - & $\mathrm{PR}, \mathrm{RS}, \mathrm{SC}$ \\
\hline \multicolumn{4}{|l|}{ Anacardiaceae } \\
\hline Schinus terebinthifolia Raddi. & - & - & AL, BA, CE, PB, PE, RN, SE, MS, ES, MG, RJ, SP, PR, RS, SC \\
\hline \multicolumn{4}{|l|}{ Apocynaceae } \\
\hline Oxypetalum tomentosum Wight ex Hook. \& Arn. & - & - & SP, PR, RS, SC \\
\hline Rhabdadenia madida (Vell.) Miers & - & - & AM, AP, PA, DF, GO, MS, MT, MG, RJ, SP, PR, RS, SC \\
\hline \multicolumn{4}{|l|}{ Araliaceae } \\
\hline Hydrocotyle bonariensis Lam. & - & - & PA, AL, BA, RN, MS, ES, MG, RJ, SP, PR, RS, SC \\
\hline \multicolumn{4}{|l|}{ Arecaceae } \\
\hline Syagrus romanzoffiana (Cham.) Glassman & - & - & BA, DF, GO, MS, ES, MG, RJ, SP, PR, RS, SC \\
\hline \multicolumn{4}{|l|}{ Asparagaceae } \\
\hline Agave americana $\mathrm{L} . * *$ & - & - & DESCONHECIDO \\
\hline Agave angustifolia Haw. ** & - & - & DESCONHECIDO \\
\hline Asparagus densiflorus (Kunth) Jessop 'Myersii'** & - & - & DESCONHECIDO \\
\hline Dracaena massangeana Rodigas ** & - & - & DESCONHECIDO \\
\hline Furcraea foetida (L.) Haw. * & - & - & BA, CE, GO, ES, MG, RJ, SP e PE, PR, SC, RS*** \\
\hline Sansevieria trifasciata Prain** & - & - & DESCONHECIDO \\
\hline Yucca gigantea Lem.** & - & - & DESCONHECIDO \\
\hline
\end{tabular}

Asteraceae

Acanthospermum australe (Loefl.) Kuntze

Achyrocline satureioides (Lam.) DC.

Acmella decumbens (Sm.) R.K.Jansen

Ambrosia artemisiifolia $\mathrm{L}$.

Baccharis crispa Spreng.

Baccharis gnaphalioides Spreng.

Baccharis retusa DC.

Baccharis spicata (Lam.) Baill.

Conyza primulifolia (Lam.) Cuatrec. \& Lourteig

Conyza sumatrensis (Retz.) E.Walker

Coreopsis lanceolata L. *

Cyrtocymura scorpioides (Mart.) Griseb.
AM, PA, RO, RR, TO, AL, BA, CE, MA, PB, PE, PI, SE, DF, GO, MS, MT, ES, MG, RJ, SP, PR, SC, RS

BA, ES, MG, RJ, SP, PR, RS, SC

RN, MS, MG, SP, PR, SC, RS

AC, AM, AP, PA, RO, BA, CE, PB, MG, RJ, SP, PR, RS, SC

BA, CE, PE, DF, GO, MS, ES, MG, RJ, SP, PR, RS, SC

$\mathrm{RS}, \mathrm{SC}$

BA, DF, GO, MT, MG, RJ, SP, PR, RS, SC

SP, PR, RS, SC

BA, ES, MG, RJ, SP, PR, RS, SC

AC, AM, PA, RO, AL, BA, PB, PE, RN, SE, DF, GO, MS, MT, ES, MG, RJ, SP, PR, RS, SC

MG, RJ, SP, PR, RS, SC

PA, AL, BA, CE, MA, PB, PE, PI, SE, DF, GO, MS, MT, ES, MG, RJ, SP, PR, RS, SC 
Tabela 1. Cont.

\begin{tabular}{|c|c|c|c|}
\hline \multirow{2}{*}{ Família/ Espécie } & \multicolumn{2}{|c|}{ Status } & \multirow{2}{*}{ Padrão de distribuição geográfico no Brasil } \\
\hline & BR & RS & \\
\hline Eclipta prostrata (L.) L. & - & - & $\begin{array}{l}\text { AC, AM, AP, PA, RO, RR, TO, AL, BA, CE, MA, PB, PE, PI, RN, } \\
\text { SE, DF, GO, MS, MT, ES, MG, RJ, SP, PR, RS, SC }\end{array}$ \\
\hline Emilia forbergii Nicolson & - & - & TODOS \\
\hline Enydra anagallis Gardner & - & - & MS, MT, RJ, SP, PR, RS, SC \\
\hline Gamochaeta americana (Mill.) Wedd. & - & - & BA, DF, MG, RJ, SP, PR, RS, SC \\
\hline Mikania involucrata Hook. \& Arn. & - & - & $\mathrm{SP}, \mathrm{PR}, \mathrm{RS}, \mathrm{SC}$ \\
\hline Noticastrum acuminatum (DC.) Cuatrec. & - & - & $\mathrm{PR}, \mathrm{RS}, \mathrm{SC}$ \\
\hline Noticastrum diffusum (Pers.) Cabrera & - & - & $\mathrm{PR}, \mathrm{RS}, \mathrm{SC}$ \\
\hline Noticastrum gnaphalioides (Baker) Cuatrec. & - & - & $\mathrm{MG}, \mathrm{SP}, \mathrm{PR}, \mathrm{RS}, \mathrm{SC}$ \\
\hline Noticastrum psammophilum (Klatt) Cuatrec. & - & $\mathrm{EN}$ & $\mathrm{RS}, \mathrm{SC}$ \\
\hline Pluchea sagittalis (Lam.) Cabrera & - & - & AC, AP, PA, BA, MA, PE, SE, GO, ES, MG, RJ, SP, PR, RS, SC \\
\hline Praxelis clematidea (Griseb.) R.M.King \& H.Rob. & - & - & $\begin{array}{l}\text { PA, TO, AL, BA, CE, PB, PE, GO, MS, MT, ES, MG, RJ, SP, PR, } \\
\text { RS, SC }\end{array}$ \\
\hline Pterocaulon lorentzii Malme & - & - & MS, MG, RJ, SP, PR, RS, SC \\
\hline Senecio crassiflorus (Poir.) De Candolle & - & - & $\mathrm{RS}, \mathrm{SC}$ \\
\hline \multicolumn{4}{|l|}{ Blechnaceae } \\
\hline $\begin{array}{l}\text { Telmatoblechnum serrulatum (Rich.) Perrie, D.J. Ohlsen } \\
\& \text { Brownsey }\end{array}$ & - & - & $\begin{array}{l}\text { AM, AP, PA, RO, RR, TO, AL, BA, MA, PB, PE, PI, RN, SE, DF, } \\
\text { GO, MS, MT, ES, MG, RJ, SP, PR, RS, SC }\end{array}$ \\
\hline \multicolumn{4}{|l|}{ Boraginaceae } \\
\hline Varronia curassavica Jacq. & - & - & $\begin{array}{l}\text { AM, AP, PA, TO, AL, BA, CE, PB, PE, PI, SE, GO, MS, ES, MG, RJ, } \\
\text { SP, PR, RS, SC }\end{array}$ \\
\hline \multicolumn{4}{|l|}{ Cactaceae } \\
\hline Nopalea cochenillifera (L.) Salm-Dyck* & - & - & AL, BA, CE, PB, PE, RN, DF, GO, ES, MG, RJ, SP, PR, SC \\
\hline Opuntia monacantha Haw. & - & - & BA, SE, ES, MG, RJ, SP, PR, RS, SC \\
\hline \multicolumn{4}{|l|}{ Calyceraceae } \\
\hline Acicarpha tribuloides Juss & - & - & $\mathrm{SP}, \mathrm{PR}, \mathrm{RS}, \mathrm{SC}$ \\
\hline \multicolumn{4}{|l|}{ Campanulaceae } \\
\hline Lobelia hederacea Cham. & - & NT & MS, MG, SP, PR, RS, SC \\
\hline Wahlenbergia linarioides (Lam.) DC. & - & - & MS, ES, MG, SP, PR, RS, SC \\
\hline \multicolumn{4}{|l|}{ Caryophyllaceae } \\
\hline Spergula arvensis L.** & - & - & DESCONHECIDO \\
\hline \multicolumn{4}{|l|}{ Casuarinaceae } \\
\hline Casuarina equisitifolia $\mathrm{L} .{ }^{* *}$ & - & - & DESCONHECIDO \\
\hline \multicolumn{4}{|l|}{ Commelinaceae } \\
\hline Callisa repens (Jacq.) L.** & - & - & DESCONHECIDO \\
\hline Commelina benghalensis L.* & - & - & $\begin{array}{l}\text { AC, AM, AP, PA, RO, RR, TO, AL, BA, CE, MA, PA, PE, PI, RN, } \\
\text { SE, DF, GO, MS, MT, ES, MG, RJ, SP, PR, RS, SC }\end{array}$ \\
\hline Commelina erecta $\mathrm{L}$. & - & - & $\begin{array}{l}\text { AM, PA, RO, TO, AL, BA, CE, MA, PB, PE, PI, RN, SE, DF, GO, } \\
\text { MS, MT, ES, MG, RJ, SP, PR, RS, SC }\end{array}$ \\
\hline Cyanotis cristata (L.) D. Don** & - & - & DESCONHECIDO \\
\hline \multicolumn{4}{|l|}{ Convolvulaceae } \\
\hline Evolvulus pusillus Choisy & - & - & MS, MT, ES, MG, RJ, SP, PR, RS, SC \\
\hline Ipomoea pes-caprae (L.) R. Br. & - & - & PA, AL, BA, CE, MA, PB, PE, PI, RN, SE, ES, RJ, SP, PR, RS, SC \\
\hline Ipomoea cairica $(\mathrm{L}$.$) Sweet$ & - & - & $\begin{array}{l}\text { RO, AL, BA, CE, PB, PE, RN, SE, DF, GO, MS, MT, ES, MG, RJ, } \\
\text { SP, PR, RS, SC }\end{array}$ \\
\hline
\end{tabular}


Tabela 1. Cont.

\begin{tabular}{|c|c|c|c|}
\hline \multirow{2}{*}{ Família/ Espécie } & \multicolumn{2}{|c|}{ Status } & \multirow{2}{*}{ Padrão de distribuição geográfico no Brasil } \\
\hline & $\mathrm{BR}$ & $\mathrm{RS}$ & \\
\hline \multicolumn{4}{|l|}{ Crassulaceae } \\
\hline Kalanchoe delagoensis Eckl. \& Zeyh.* & - & - & ES, MG, RJ, SP, PR, SC \\
\hline Kalanchoe fedtschenkoi Raym.-Hamet \& H. Perrier* & - & - & $\mathrm{ES}, \mathrm{MG}, \mathrm{RS}^{* * * *}$ \\
\hline Kalanchoe pinnata (Lam.) Pers.* & - & - & AC, BA, CE, PB, DF, MS, MT, ES, MG, RJ, SP, PR, RS, SC \\
\hline \multicolumn{4}{|l|}{ Cyperaceae } \\
\hline Androtrichum trigynum (Spreng.) H. Pfeiff. & - & - & RJ, SP, PR, RS, SC \\
\hline Cyperus aggregatus (Willd.) & - & - & TODOS \\
\hline Cyperus odoratus L. & - & - & TODOS \\
\hline Cyperus sesquiflorus (Torr.) Mattf. \& Kük. & - & - & $\begin{array}{l}\text { AC, AM, AP, PA, RO, RR, TO, AL, BA, CE, MA, PB, PE, PI, RN, } \\
\text { SE, DF, GO, MS, MT, ES, MG, RJ, SP, RS }\end{array}$ \\
\hline Cyperus obtusatus (J.Presl \& C.Presl) Mattf. \& Kük. & - & - & TODOS \\
\hline \multicolumn{4}{|l|}{ Dryopteridaceae } \\
\hline Rumohra adiantiformis (G. Forst.) Ching & - & - & AL, BA, CE, PE, DF, ES, MG, RJ, SP, PR, RS, SC \\
\hline \multicolumn{4}{|l|}{ Equisetaceae } \\
\hline Equisetum giganteum $\mathrm{L}$. & - & - & RO, BA, PB, PE, PI, DF, GO, MS, MT, ES, MG, RJ, SP, PR, RS, SC \\
\hline \multicolumn{4}{|l|}{ Euphorbiaceae } \\
\hline Croton lundianus (Didr.) Müll.Arg. & - & - & $\begin{array}{l}\text { AM, PA, RO, TO, AL, BA, CE, MA, PE, PI, SE, GO, MS, MT, ES, } \\
\text { MG, RJ, SP, PR, SC, RS }\end{array}$ \\
\hline Ricinus communis L.** & - & - & DESCONHECIDO \\
\hline Sapium glandulosum (L.) Morong & - & - & TODOS \\
\hline \multicolumn{4}{|l|}{ Fabaceae } \\
\hline Desmodium adscendens (Sw.) DC. * & - & - & $\begin{array}{l}\text { AC, AM, AP, PA, RO, RR, TO, AL, BA, CE, MA, PB, PE, PI, RN, } \\
\text { SE, GO, ES, MG, RJ, SP, PR, RS, SC }\end{array}$ \\
\hline Desmodium incanum (Sw.) DC. * & - & - & TODOS \\
\hline Mimosa bimucronata (DC.) Kuntze & - & - & AL, BA, CE, MA, PE, SE, DF, GO, MS, ES, MG, RJ, SP, PR, RS, SC \\
\hline $\begin{array}{l}\text { Senna macranthera (DC. ex Collad.) H.S.Irwin \& Bar- } \\
\text { by }\end{array}$ & - & - & $\begin{array}{l}\text { TO, AL, BA, CE, PB, PE, PI, RN, DF, GO, MT, ES, MG, RJ, SP, PR, } \\
\text { RS }\end{array}$ \\
\hline Vigna luteola (Jacq.) Benth. & - & - & PA, BA, PI, MG, RJ, SP, PR, RS, SC \\
\hline \multicolumn{4}{|l|}{ Geraniaceae } \\
\hline Pelargonium zonale (L.) L'Hér** & - & - & DESCONHECIDO \\
\hline \multicolumn{4}{|l|}{ Iridaceae } \\
\hline Crocosmia crocosmiiflora (Lemoine) N.E.Br* & - & - & BA, DF, ES, MG, RJ, SP, PR, RS, SC \\
\hline \multicolumn{4}{|l|}{ Juncaceae } \\
\hline Juncus microcephalus Kunth. & - & - & BA, GO, MG, RJ, SP, PR, RS, SC \\
\hline \multicolumn{4}{|l|}{ Lamiaceae } \\
\hline Hyptis lagenaria A.St.-Hil. ex Benth. & - & - & MG, SP, PR, RS, SC \\
\hline Plectranthus neochilus Schltr.** & - & - & DESCONHECIDO \\
\hline \multicolumn{4}{|l|}{ Lycopodiaceae } \\
\hline Lycopodiella longipes (Grev. \& Hooker) Holub & - & - & AC, AM, RR, BA, DF, GO, MG, RJ, SP, PR, RS, SC \\
\hline \multicolumn{4}{|l|}{ Malvaceae } \\
\hline Hibiscus diversifolius Jacq. & - & - & ES, RJ, SP, PR, RS, SC \\
\hline \multicolumn{4}{|l|}{ Melastomataceae } \\
\hline Pleroma asperior (Cham.) Triana & $\mathrm{EN}$ & - & $\mathrm{SC}, \mathrm{RS}$ \\
\hline Tibouchina versicolor (Lindl.) Cogn. & - & - & SP,PR, RS, SC \\
\hline
\end{tabular}


Tabela 1. Cont.

\begin{tabular}{|c|c|c|c|}
\hline \multirow{2}{*}{ Família/ Espécie } & \multicolumn{2}{|c|}{ Status } & \multirow{2}{*}{ Padrão de distribuição geográfico no Brasil } \\
\hline & BR & RS & \\
\hline Musa paradisiaca L.** & - & - & DESCONHECIDO \\
\hline \multicolumn{4}{|l|}{ Myrtaceae } \\
\hline Eugenia uniflora L. & - & - & BA, MS, ES, MG, RJ, SP, PR, RS, SC \\
\hline Psidium cattleyanum Sabine & - & - & BA, CE, PE, SE, ES, MG, RJ, SP, PR, RS, SC \\
\hline Psidium guajava L.* & - & - & $\begin{array}{l}\text { AC, AM, AL, BA, CE, MA, PE, PI, SE, MS, MT, ES, MG, RJ, SP, } \\
\text { PR, RS, SC }\end{array}$ \\
\hline Syzygium cumini (L.) Skeels* & - & - & AM, RR, BA, PE, ES, MG, RJ, SP, PR, RS, SC \\
\hline \multicolumn{4}{|l|}{ Onagraceae } \\
\hline Ludwigia multinervia (Hook. \& Arn.) Ramamoorthy & - & - & $\mathrm{SC}, \mathrm{RS}$ \\
\hline Oenothera mollisima L. & - & - & $\mathrm{SC}, \mathrm{RS}$ \\
\hline \multicolumn{4}{|l|}{ Orchidaceae } \\
\hline Epidendrum fulgens Brongn & - & - & $\mathrm{RJ}, \mathrm{SP}, \mathrm{PR}, \mathrm{RS}, \mathrm{SC}$ \\
\hline Habenaria parviflora Lindl. & - & - & RR, BA, SE, DF, GO, ES, MG, RJ, SP, PR, RS, SC \\
\hline \multicolumn{4}{|l|}{ Oxalidaceae } \\
\hline Oxalis bipartita A.St.-Hil. & - & - & $\mathrm{MG}, \mathrm{PR}, \mathrm{RS}, \mathrm{SC}$ \\
\hline \multicolumn{4}{|l|}{ Passifloraceae } \\
\hline Passiflora caerulea L. & - & - & AL, BA, CE, PB, PE, RN, SE, ES, MG, RJ, SP, PR, RS, SC \\
\hline \multicolumn{4}{|l|}{ Plantaginaceae } \\
\hline Bacopa monnieri (L.) Penell & - & - & BA, CE, PE, ES, RJ, SP, PR, RS, SC \\
\hline Plantago australis Lam. & - & - & MG, RJ, SP, PR, RS, SC \\
\hline \multicolumn{4}{|l|}{ Poaceae } \\
\hline Andropogon arenarius Hack. & - & - & SP, PR, RS, SC \\
\hline Andropogon selloanus Hack. & - & - & $\begin{array}{l}\text { AM, PA, RR, TO, BA, CE, MA, PB, PE, PI, RN, SE, DF, GO, MS, } \\
\text { MT, ES, MG, RJ, SP, PR, RS, SC }\end{array}$ \\
\hline Cenchrus incertus Curt. & - & - & $\mathrm{RS}, \mathrm{SC}$ \\
\hline Chascolytrum subaristatum (Lam.) Desv. & - & - & RS, PR \\
\hline Dichantelium sabulorum (Lam.) Gould \& C.A. Clark & - & - & PB, MG, RJ, SP, PR, RS, SC \\
\hline Digitaria eriantha Steud. * & - & - & PE, MS, MG, SP, PR, RS, SC \\
\hline Digitaria sanguinalis (L.) Scop** & - & - & DESCONHECIDO \\
\hline Eragrostis trichocolea Hack. \& Arechav. & - & - & SP, PR, RS, SC \\
\hline Imperata brasiliensis Trin. & - & - & AP, BA, MA, DF, GO, MS, MT, ES, MG, RJ, SP, PR, RS, SC \\
\hline Panicum racemosum (P. Beauv.) Spreng. & - & - & BA, CE, MA, PE, RN, ES, RJ, SP, RS, SC \\
\hline Panicum repens L. * & - & - & AM, PA, AL, BA, PE, RN, MS, MT, ES, MG, SP, PR, RS, SC \\
\hline Setaria parviflora (Poir.) Kerguélen & - & & $\begin{array}{l}\text { AC, AM, AP, PA, RR, TO, AL, BA, MA, PB, PE, PI, RN, SE, DF, } \\
\text { GO, MS, MT, ES, MG, RJ, SP, PR, RS, SC }\end{array}$ \\
\hline Spartina ciliata Brongn & - & - & $\mathrm{RJ}, \mathrm{SP}, \mathrm{PR}, \mathrm{RS}, \mathrm{SC}$ \\
\hline Uroclhoa decumbes (Stapf) R. D. Webster* & - & - & $\begin{array}{l}\text { PA, RO, RR, TO, AL, BA, CE, PB, PE, PI, RN, SE, DF, GO, MS, } \\
\text { MT, ES, MG, SP, PR, RS, SC }\end{array}$ \\
\hline Zea mays L.** & - & - & DESCONHECIDO \\
\hline \multicolumn{4}{|l|}{ Polygalaceae } \\
\hline Polygala cyparissias A.St.-Hil. \& Moq. & - & - & AL, BA, CE, PB, PE, SE, ES, RJ, SP, PR, RS, SC \\
\hline Polygonaceae & & & \\
\hline
\end{tabular}


Tabela 1. Cont.

\begin{tabular}{|c|c|c|c|}
\hline \multirow{2}{*}{ Família/ Espécie } & \multicolumn{2}{|c|}{ Status } & \multirow{2}{*}{ Padrão de distribuição geográfico no Brasil } \\
\hline & $\mathrm{BR}$ & RS & \\
\hline Polygonum acuminatum Kunth. & - & - & $\begin{array}{l}\text { AC, AM, AP, PA, RO, RR, TO, AL, BA, CE, MA, PE, RN, SE, DF, } \\
\text { GO, MS, MT, ES, MG, RJ, SP, PR, RS, SC }\end{array}$ \\
\hline Polygonum punctatum Elliot & - & - & $\begin{array}{l}\text { AC, AM, PA, RO, AL, BA, CE, MA, PB, PE, PI, SE, DF, GO, MS, } \\
\text { MT, ES, MG, RJ, SP, PR, RS, SC }\end{array}$ \\
\hline
\end{tabular}

Polypodiaceae

\begin{tabular}{|c|c|c|c|}
\hline Pleopeltis lepidopteris (Langsd. \& Fisch.) de la Sota & - & - & ES, RJ, SP, PR, RS, SC \\
\hline \multicolumn{4}{|l|}{ Portulacaceae } \\
\hline Portulaca grandiflora Hook. & - & - & BA, GO, MS, MT, MG, SP, PR, RS \\
\hline \multicolumn{4}{|l|}{ Primulaceae } \\
\hline Myrsine coriacea (Sw.) R.Br. ex Roem. \& Schult. & - & - & BA, PE, DF, GO, MS, MT, ES, MG, RJ, SP, PR, RS, SC \\
\hline \multicolumn{4}{|l|}{ Rosaceae } \\
\hline Margyricarpus pinnatus (Lam.) Kuntze & - & - & MS, PR, RS, SC \\
\hline \multicolumn{4}{|l|}{ Rubiaceae } \\
\hline Diodia saponariifolia (Cham. \& Schltdl.) K.Schum. & - & - & BA, GO, MS, MT, ES, MG, RJ, SP, PR, RS, SC \\
\hline Hamelia patens Jacq.** & - & - & DESCONHECIDO \\
\hline $\begin{array}{l}\text { Hexasepalum apiculatum (Willd.) Delprete \& J.H. } \\
\text { Kirkbr. }\end{array}$ & - & - & $\begin{array}{l}\text { AP, RO, TO, AL, BA, CE, MA, PB, PE, PI, RN, SE, DF, GO, MS, } \\
\text { MT, ES, MG, RJ, SP, PR, RS, SC }\end{array}$ \\
\hline Richardia brasiliensis Gomes & - & - & $\begin{array}{l}\text { A, TO, AL, BA, CE, PB, PE, PI, RN, DF, GO, MS, MT, ES, MG, RJ, } \\
\text { SP, PR, RS, SC }\end{array}$ \\
\hline \multicolumn{4}{|l|}{ Sapindaceae } \\
\hline Dodonaea viscosa Jacq. & - & - & PA, RO, AL, BA, CE, MA, PB, PE, RN, ES, MG, RJ, SP, PR, RS, SC \\
\hline \multicolumn{4}{|l|}{ Smilacaceae } \\
\hline Smilax campestris Griseb. & - & - & TO, BA, CE, PE, DF, MS, MG, RJ, SP, PR, RS, SC \\
\hline \multicolumn{4}{|l|}{ Solanaceae } \\
\hline Petunia integrifolia (Hook.) Schinz \& Thell. & - & - & $\mathrm{RS}, \mathrm{SC}$ \\
\hline Salpichroa origanifolia (Lam.) Baill. & - & - & $\mathrm{RS}, \mathrm{SC}$ \\
\hline Solanum americanum Mill. & - & - & TODOS \\
\hline Solanum commersonii Dunal & - & - & $\mathrm{PR}, \mathrm{SC}, \mathrm{RS}$ \\
\hline Solanum reineckii Briq. & - & - & $\mathrm{RS}, \mathrm{SC}$ \\
\hline Solanum sisymbriifolium Lam. & - & - & AC, RO, BA, DF, GO, MS, MT, ES, MG, RJ, SP, PR, RS, SC \\
\hline \multicolumn{4}{|l|}{ Thelypteridaceae } \\
\hline Cyclosorus interruptus (Willd.) H. Ito & - & - & $\begin{array}{l}\text { AM, AP, AL, BA, CE, MA, PB, PE, DF, GO, MS, MT, ES, MG, RJ, } \\
\text { SP, PR, RS, SC }\end{array}$ \\
\hline \multicolumn{4}{|l|}{ Thymelaeaceae } \\
\hline Daphnopsis racemosa (Sw.) R.Br. ex Roem. \& Schult. & - & - & BA, PE, MS, MT, ES, MG, RJ, SP, PR, RS, SC \\
\hline \multicolumn{4}{|l|}{ Verbenaceae } \\
\hline Glandularia aristigera (S.Moore) Tronc. & - & - & MS, SP, PR, RS, SC \\
\hline Lantana camara L. * & - & - & $\begin{array}{l}\text { AC, AM, AP, PA, RO, RO, TO, BA, CE, MA, PA, PE, PI, RN, DF, } \\
\text { GO, MS, MT, ES, MG, RJ, SP, PR, RS, SC }\end{array}$ \\
\hline Lippia alba (Mill.) N.E.Br. ex P. Wilson & - & - & $\begin{array}{l}\text { AC, AM, AP, PA, RO, RR, TO, AL, BA, CE, MA, PB, PE, PI, RN, } \\
\text { SE, DF, GO, MS, MT, ES, MG, RJ, SP, PR, RS, SC }\end{array}$ \\
\hline Stachytarpheta cayennsis (Rich.) Vahl & - & - & $\begin{array}{l}\text { AC, AM, AP, PA, RO, TO, BA, CE, MA, PE, SE, DF, GO, MS, MT, } \\
\text { ES, MG, RJ, SP, PR, RS, SC }\end{array}$ \\
\hline
\end{tabular}


Tabela 1. Cont.

\begin{tabular}{llll}
\hline Família/ Espécie & \multicolumn{2}{c}{ Status } & Padrão de distribuição geográfico no Brasil \\
\cline { 2 - 3 } Vitaceae & $\mathrm{BR} \quad \mathrm{RS}$ & TODOS \\
\hline Cissus verticillata (L.) Nicolson \& C.E.Jarvis & - & - & \\
\hline Xanthorrhoeaceae & - & - & DESCONHECIDO
\end{tabular}

O número de espécies identificado no presente estudo (141) é maior do que o encontrado em outros estudos realizados em áreas de restinga no sul do Brasil. Como por exemplo, Cordazzo \& Seeliger (1987), que identificaram 66 espécies em uma área de dunas costeiras, próxima a Reserva Ecológica do Taim, no Litoral Sul do Estado do Rio Grande do Sul, Klein et al. (2007), que identificaram 60 espécies em uma restinga herbácea em Araranguá, Santa Catarina, Palma \& Jarenkow (2008), que registraram 31 espécies nas dunas costeiras da Praia da Itapeva, em Torres, Rio Grande do Sul, e Schlickmann et al. (2016) que registraram 15 espécies para o Morro dos Conventos, Santa Catarina.

Waechter $(1985,1998)$ e Scherer (2009) observaram um expressivo gradiente latitudinal de diversidade, caracterizado pela diminuição da riqueza florística no sentido norte-sul, ocasionado principalmente pela descontinuidade climática ocorrente no paralelo $30^{\circ} \mathrm{Sul}$, onde ocorre a transição entre os domínios fitogeográficos da Mata Atlântica e Pampa (Fischi \& Pirani 2009). Embora as restingas pareçam muito semelhantes, além das variações florísticas entre as comunidades nas diferentes faixas latitudinais da planície, também ocorrem variações no sentindo leste-oeste, marcadas basicamente pela sucessão das comunidades em virtude de maior estabilidade edáfica e menor influência marinha (Rambo 1954). No entanto, a diferença entre a riqueza de espécies entre os estudos citados anteriormente pode ser atribuída à metodologia e o esforço amostral empregado, bem como à heterogeneidade ambiental das dunas de Torres.

$\mathrm{Na}$ análise fitossociológica, a área sem cobertura vegetal totalizou $40,6 \%$ da área amostrada, sendo que apenas três unidades amostrais apresentaram nenhuma cobertura vegetal. A cobertura vegetal total foi de $60,4 \%$ na área amostrada. Esse valor médio de cobertura vegetal corresponde ao estágio III da classificação morfoecológica de dunas apresentada por Calliari et al. (2005) que representa $40 \%$ das dunas do litoral norte. Estes dados também são corroborados por Palma \& Jarenkow (2008) que encontraram quatro unidades amostrais sem cobertura ao longo do ano. Palma \& Jarenkow (2008), também destacam que a comunidade vegetal em dunas costeiras possui geralmente uma estrutura oligárquica caracterizada pela alta dominância de poucas espécies, representadas nas dunas primárias por Blutaparon portulacoides (St.-Hil.) Mears e Paspalum vaginatum Sw., nas dunas secundárias e terciárias por Hydrocotyle bonariensis Lam. Mesmo padrão também foi observado no presente estudo, bem como por Cordazzo \& Costa (1989) e Henriques et al. (1984).

Foram identificadas 33 espécies, pertencentes a 16 famílias (Tab. 2). As espécies que apresentaram os valores de importância (VI) mais elevados foram Panicum racemosum (P. Beauv.) Spreng. (FR: 16,43\%, CR: 28,47\%, VI: 22,45\%), seguido por Panicum repens L. (FR: $8,57 \%$, $\mathrm{CR}_{\mathrm{i}}: 11,71 \%$, VI: 10,14\%), H. bonariensis (FR: $11,43 \%$, $\mathrm{CR}_{\mathrm{i}}$ : 6,66\%, VI: 9,04\%), Andropogon arenarius Hack. (FR: 3,57\%, CR: 9,54\%, VI: 6,56\%) e Cenchrus incertus Curt. (FR: $5,72 \%, \mathrm{CR}_{\mathrm{i}}: 5,76 \%$, VI: 5,74\%). Os dados apresentados aqui corroboram os já encontrados por Klein et al. (2007), Palma \& Jarenkow (2008) e Schlickmann et al. (2016) que também citaram $P$. racemosum, $H$. bonariensis e $A$. arenarius entre as espécies com maior valor de importância. Palma \& Jarenkow (2008) também encontraram Senecio crassifolius (Poir.) De Candolle como uma das espécies com maior valor de importância, o que não foi corroborado pelo presente estudo. Por outro lado, os demais estudos não citaram $C$. incertus, o qual obteve elevado valor de importância na área deste estudo.

É interessante notar que a maioria das espécies com os maiores valores de importância são rizomatosas, como por exemplo $P$. racemosum, $H$. bonariensis e $P$. repens. Esta forma de crescimento, altamente adaptada para a reprodução vegetativa e para a ocupação rápida de ambientes, atua de forma significativa nos processos de fixação das dunas (Waechter 1985, Cordazzo \& Seeliger 1995, Brack 2009). Desta forma, garantem o processo de sucessão no sentido oceano continente, bem como evitam o avanço do mar e das dunas móveis, que por vezes acabam danificando construções humanas junto à praia.

A espécie $P$. racemosum, que obteve o maior valor de importância, destaca-se pela alta capacidade de fixação de dunas e por suportar soterramento de grandes quantidades de areia (Waechter 1985). Esta espécie apresenta distribuição cosmopolita pelos trópicos e subtrópicos, em campos abertos e solos arenosos do litoral (Zuloaga et al. 2001), sendo abundante no extremo sul do Brasil (Cordazzo \& Seeliger 1995). As populações de $P$. racemosum ocupam áreas onde o substrato não é estável e há variação no acúmulo de areia e são mantidas, principalmente, pelo crescimento vegetativo, pois apresentam pouca floração e baixa viabilidade das sementes devido à dessecação pelo vento, altas temperaturas, abrasão da areia e predação dos propágulos por besouros (Costa et al. 1991). 
Tabela 2. Parâmetros fitossociológicos estimados para vegetação das dunas costeiras de Torres, Rio Grande do Sul. UA $\mathrm{A}_{\mathrm{i}}=$ número de unidades amostrais, $\mathrm{FA}_{\mathrm{i}}=$ frequência absoluta $(\%), \mathrm{FR}_{\mathrm{i}}=$ frequência relativa $(\%), \mathrm{CA}_{\mathrm{i}}=$ cobertura absoluta $(\%), \mathrm{CR}_{\mathrm{i}}=$ cobertura relativa $(\%)$ e $\mathrm{VI}=$ valor de importância (\%). * Espécie naturalizada.

\begin{tabular}{|c|c|c|c|c|c|c|}
\hline Espécies & $\mathbf{U A}_{\mathrm{i}}$ & $\mathbf{F A}_{\mathrm{i}}$ & $\mathbf{F R}_{\mathbf{i}}$ & $\mathbf{C A}_{\mathrm{i}}$ & $\mathbf{C R}_{\mathrm{i}}$ & VI \\
\hline Panicum racemosum (P. Beauv.) Spreng. & 23 & 51,11 & 16,43 & 761 & 28,47 & 22,45 \\
\hline Panicum repens $\mathrm{L} . *$ & 12 & 26,67 & 8,57 & 313 & 11,71 & 10,14 \\
\hline Hydrocotyle bonariensis Lam. & 16 & 35,56 & 11,43 & 178 & 6,66 & 9,04 \\
\hline Andropogon arenarius Hack. & 5 & 11,11 & 3,57 & 255 & 9,54 & 6,56 \\
\hline Cenchrus incertus Curt. & 8 & 17,78 & 5,72 & 154 & 5,76 & 5,74 \\
\hline Andropogon selloanus Hack. & 5 & 11,11 & 3,57 & 185 & 6,92 & 5,25 \\
\hline Ipomoea pes-caprae (L.) R. Br. & 4 & 8,89 & 2,86 & 102 & 3,82 & 3,34 \\
\hline Oxypetalum tomentosum Wight ex Hook. \& Arn. & 6 & 13,33 & 4,28 & 62 & 2,32 & 3,30 \\
\hline Gamochaeta americana (Mill.) Wedd. & 6 & 13,33 & 4,29 & 51 & 1,91 & 3,10 \\
\hline Senecio crassiflorus (Poir.) De Candolle & 5 & 11,11 & 3,57 & 50 & 1,87 & 2,72 \\
\hline Eragrostis trichocolea Hack. \& Arechav. & 4 & 8,89 & 2,86 & 67 & 2,51 & 2,68 \\
\hline Androtrichum trigynum (Spreng.) H. Pfeiff. & 3 & 6,67 & 2,14 & 60 & 2,24 & 2,19 \\
\hline Spartina ciliata Brongn & 2 & 4,44 & 1,43 & 70 & 2,62 & 2,02 \\
\hline Chascolytrum subaristatum (Lam.) Desv. & 4 & 8,89 & 2,86 & 26 & 0,97 & 1,91 \\
\hline Conyza sumatrensis (Retz.) E.Walker & 3 & 6,67 & 2,14 & 37 & 1,38 & 1,76 \\
\hline Blutaparon portulacoides (St.-Hil.) Mears & 3 & 6,67 & 2,14 & 28 & 1,05 & 1,60 \\
\hline Glandularia aristigera (S.Moore) Tronc. & 3 & 6,67 & 2,14 & 25 & 0,94 & 1,54 \\
\hline Acmella sp. & 3 & 6,67 & 2,14 & 22 & 0,82 & 1,48 \\
\hline Paspalum sp. & 2 & 4,44 & 1,43 & 40 & 1,50 & 1,46 \\
\hline Noticastrum acuminatum (DC.) Cuatrec. & 2 & 4,44 & 1,43 & 35 & 1,31 & 1,37 \\
\hline Varronia curassavica Jacq. & 3 & 6,67 & 2,14 & 15 & 0,56 & 1,35 \\
\hline Polygala cyparissias A.St.-Hil. \& Moq. & 3 & 6,67 & 2,14 & 12 & 0,45 & 1,30 \\
\hline Desmodium incanum Sw. (DC.) * & 2 & 4,44 & 1,43 & 20 & 0,75 & 1,09 \\
\hline Panicum sp. & 2 & 4,44 & 1,43 & 20 & 0,75 & 1,09 \\
\hline Carpobrotus edulis (L) N.E.Br. * & 1 & 2,22 & 0,71 & 35 & 1,31 & 1,01 \\
\hline Margyricarpus pinnatus (Lam.) Kuntze & 2 & 4,44 & 1,43 & 8 & 0,30 & 0,86 \\
\hline Baccharis crispa Spreng. & 1 & 2,22 & 0,71 & 25 & 0,94 & 0,82 \\
\hline Pterocaulon lorentzii Malme & 2 & 4,44 & 1,43 & 3 & 0,11 & 0,77 \\
\hline Solanum reineckii Briq. & 1 & 2,22 & 0,71 & 5 & 0,19 & 0,45 \\
\hline Pleopeltis lepidopteris Langsd. \& Fisch. & 1 & 2,22 & 0,71 & 3 & 0,11 & 0,41 \\
\hline Acicarpha tribuloides Juss. & 1 & 2,22 & 0,71 & 2 & 0,07 & 0,39 \\
\hline Cyperus aggregatus (Willd.) & 1 & 2,22 & 0,71 & 2 & 0,07 & 0,39 \\
\hline Pluchea sagittalis (Lam.) Cabrera & 1 & 2,22 & 0,71 & 2 & 0,07 & 0,39 \\
\hline Totais & 140 & 311,11 & 100 & 2673 & 100 & 100 \\
\hline
\end{tabular}

As espécies com valor de importância mais baixo, ou seja, menor do que 0,5 foram Solanum reineckii Briq., Pleopeltis lepidopteris Langsd. \& Fisch., Acicarpha tribuloides Juss., Cyperus aggregatus (Willd.) e Pluchea sagittalis (Lam.) Cabrera, o que não corrobora os dados de Palma \& Jarenkow (2008) que encontraram Cyperus obtusatus (J.Presl \& C.Presl) Mattf. \& Kük, Gamochaeta americana (Mill.) Wedd., P. vaginatum e Digitaria aequiglumis (Hack. \& Arechav.) Parodi.

$\mathrm{Na}$ área foram observadas, além das comunidades vegetais características das dunas costeiras, uma grande variedade de espécies em ambientes alterados próximos aos canais de drenagem, acessos à praia e ao passeio público.
Isto se deve, provavelmente, ao aporte de nutrientes por ação antrópica que beneficia a ocorrência e desenvolvimento de outras espécies nativas ou exóticas, divergindo da limitação imposta pelos solos de restinga, que restringe a ocorrência de muitas espécies pelas condições estressantes como a falta de nutrientes (Rambo 1956). Pelo observado nas atividades de campo, o conjunto de espécies que habita estes ambientes são de caráter pioneiro, ruderal ou então exóticas naturalizadas, como Eclipta prostrata (L.) L., Enydra anagallis Gardner, Callisa repens (Jacq.) L., Oenothera mollisima L. e Diodia saponarifolia (Cham. \& Schltdl.) K.Schum. 
Os ambientes perturbados favorecem o aparecimento de espécies exóticas (Williamson 1996, Parker \& Reichard 1997, Ziller 2001), as quais quando adaptadas aos ecossistemas dunares, provocam a perda das características exclusivas da vegetação das dunas, resultando em comunidades totalmente diferentes das originais (Rosa \& Cordazzo 2007). Das 37 espécies exóticas encontradas no levantamento florístico, oito são consideradas invasoras (Rio Grande do Sul 2013), 17 são naturalizadas e três cultivadas (Flora do Brasil 2020). Na análise fitossociológica, foram registradas apenas três espécies exóticas, classificadas como naturalizadas: $P$. repens, Desmodium incanum $\mathrm{Sw}$. (DC.) e Carpobrotus edulis (L.) N. E. Br.

Em relação às espécies identificadas como invasoras de categoria I, ou seja, aquelas que de acordo com Rio Grande do Sul (2013) apresentam proibição para qualquer tipo de manejo, foram identificadas Furcraea foetida (L.) Haw., Casuarina equisitifolia L., Kalanchoe pinnata (Lam.) Pers., Crocosmia crocosmiiflora (Lemoine) N.E.Br, Psidium guajava L. Para a categoria II, ou seja, que podem ser utilizadas em condições controladas (Rio Grande do Sul 2013), registrou-se a presença de três espécies: Sansevieria trifasciata Prain, Syzygium cumini (L.) Skeels e Uroclhoa decumbes (Stapf) R.D.Webster.

A espécie C. equisetifolia é arbórea, tolerante às condições de salinidade, aridez e também à baixa fertilidade do solo, além disso, apresenta sementes pequenas, produzidas em alta quantidade, dispersão pelo vento, alta longevidade das sementes no solo, capacidade de germinar em ampla faixa de temperatura e luminosidade (Zimmermann 2016). A espécie F. foetida, mais conhecida como "piteira", possui valor ornamental e propaga-se facilmente através dos numerosos bulbilhos que se formam após o desenvolvimento das flores (Lorenzi 2013). Ambas se adaptam a áreas degradadas e possuem eficiência no sucesso reprodutivo (Hueck 1953, Francis 2009).

De acordo com Matos \& Pivelo (2009), as espécies exóticas naturalizadas caracterizam-se pela capacidade de formar populações persistentes, mas sem causar prejuízos à comunidade nativa. Entre as espécies consideradas naturalizadas, $C$. edulis, mais conhecido como chorãoda-praia, destacou-se na área de estudo pela presença em 29 pontos, formando extensos tapetes, principalmente nas dunas próximas ao calçadão. De origem sul-africana, apresenta grande valor ornamental, por suas flores cor rosa e muito vistosas e é considerada invasora em diversos locais da Oceania, Europa e regiões subtropicais das Américas (Flora do Brasil 2020).

Já as espécies cultivadas são aquelas espécies domesticadas, que foram criadas ou melhoradas para o cultivo (Barbieri \& Stumpf 2008), sendo registradas Riccinus comunis L., Musa paradisiaca L. e Zea mays L. A mamona, $R$. comunis, proveniente da África tropical, foi amplamente introduzida com intuito de ornamentação de jardins (Matthews 2005). A espécie M. paradisíaca destaca-se como uma das frutas mais comercializadas mundialmente, e o milho, Z. mays, é uma das espécies que compõem a base alimentar da humanidade (Lorenzi \& Souza 2012). A reprodução vegetativa, frequente em bananeiras (Borges \& Souza 2004), pode favorecer a dispersão de espécies exóticas, através de restos de podas e jardinagens (Rosa \& Cordazzo 2007).

Para Schneider (2007), as espécies exóticas são encontradas nos mais diversos locais e estão intimamente relacionadas à presença humana, evidenciando como um dos principais impactos antrópicos, a introdução de espécies exóticas pelos moradores da cidade com intuito de obter sombra, ornamentação, fixação de dunas, ou até mesmo sem intenção de cultivo através dos restos de podas e galhos, sementes e restos de frutas que são lançados ao solo.

Restos de podas e jardinagens foram observados nas dunas da área de estudo, além de outros impactos antrópicos como pisoteio demasiado, disposição de resíduos sólidos, abertura de trilhas e passarelas para acesso à praia, instalação de quiosques sobre as dunas próximas ao calçadão. Essas perturbações, também verificadas em um trabalho de campo realizado por Rosa \& Cordazzo (2007), sobre a vegetação nas dunas da Praia do Cassino (RS), impactam as dunas, tornando o ambiente frágil e favorável para entrada de espécies exóticas que podem ocupar o ambiente eliminando as espécies nativas, especialmente, espécies ameaçadas de extinção.

De acordo com Rio Grande do Sul (2014), três espécies ocorrentes na área de estudo estão classificadas em diferentes categorias de ameaça para o estado do Rio Grande do Sul: Pleroma asperior (Cham.) Triana (EN), B. portulacoides (VU) e Gomphrena perennis L. (NT). A espécie $P$. asperior é endêmica do Brasil, com ocorrência confirmada apenas para o estado do RS (Flora do Brasil 2020). Apresenta o status de conservação em perigo devido à redução de população projetada e observada em mais de 50\% (Rio Grande do Sul 2014). No presente estudo, a espécie foi registrada apenas durante o caminhamento, não havendo parâmetros fitossociológicos para análise. A espécie B. portulacoides, característica das dunas frontais (Waechter 1985, Valduga et al. 2013), apresenta o status de conservação vulnerável devido à redução de população em mais de 50\%, estimada através de observação direta e análise do índice de abundância apropriado para o táxon (Rio Grande do Sul 2014). Na análise fitossociológica do presente estudo, B. portulacoides apresenta: VI 1,60\%, $\mathrm{FR}_{\mathrm{i}} 2,14 \%, \mathrm{CR}_{\mathrm{i}} 1,05 \%$. A espécie G. perennis ocorre em dunas ou campos secos da região sul do Brasil (PR, SC e RS) além de outros países como Argentina, Bolívia, Equador, Paraguai e Uruguai (Siqueira 1992). Apresenta o status de conservação como quase ameaçada devido a sua distribuição geográfica restrita (Rio Grande do Sul 2014). Foi registrada apenas durante o caminhamento, não havendo parâmetros fitossociológicos para análise.

Outras três espécies estão citadas pela Lista da Flora Brasileira ameaçada de extinção (Brasil 2014): Lobelia hederacea Cham. (NT), Noticastrum psammophilum (Klatt) Cuatrec. (EN) e S. crassiflorus (NT). Estas espécies não estão ameaçadas no estado do RS, porém aparecem na 
lista nacional. A espécie N. psammophilum habita áreas de dunas e restingas do sul do Brasil, é polinizada por insetos e dispersada pelo vento. Seu status de conservação está em perigo devido a sua distribuição geográfica restrita, considerando que as áreas de ocorrência estão severamente fragmentadas e em declínio (CNCFlora 2017). Esta espécie também foi verificada apenas no caminhamento, não havendo parâmetros fitossociológicos para análise. A espécie $S$. crassiflorus é uma espécie característica das dunas secundárias e terciárias (Waechter 1985), polinizada por insetos e dispersada pelo vento. Apresenta o status de conservação como quase ameaçada, mas sem identificação dos critérios (CNCFlora 2017). Na área de estudo, foi verificada em cinco unidades amostrais de três transectos, obtendo valor de importância relativamente alto $\left(\mathrm{FR}_{\mathrm{i}}\right.$ $3,57 \%, \mathrm{CR}_{\mathrm{i}} 1,87 \%$, VI $2,72 \%$ ), além de ter sido amplamente observada durante o caminhamento.

Os critérios de classificação do status de ameaça das espécies citadas acima reforçam a fragilidade das Áreas de Formação Pioneiras como as dunas, as quais encontram-se consideravelmente alteradas ou até mesmo suprimidas em alguns locais (Duarte \& Bencke 2006). A ocorrência de espécies ameaçadas comprova a importância dos estudos florísticos como subsídio para estudos posteriores que abordem ações de conservação e recuperação das formações vegetais (Freitas \& Magalhães 2012), harmonizando o uso desta área e a conservação da biodiversidade existente nela para preservar as espécies ameaçadas, bem como as funções ecológicas desempenhadas por este ambiente.

Apesar de serem ecossistemas de grande beleza paisagística, terem alta importância natural por abrigar elevada diversidade biológica e áreas de nidificação de diversas espécies de aves, as dunas costeiras estão se tornando fragilizadas, principalmente pela influência antrópica. A interferência humana para expansão urbana, exploração de areia, drenagem de banhados, pisoteio de vegetação, tráfego de veículos, plantio de espécies exóticas, descarte de resíduos sólidos e efluentes líquidos são algumas das ações que têm se mostrado nocivas aos recursos naturais deste ambiente (Waechter 1985, Brack 2009). Torna-se imprescindível, portanto, planejar medidas que visem o controle das populações de espécies exóticas estabelecidas e prevenção de novos focos, juntamente com a construção de conhecimento sobre a composição florística das espécies nas dunas costeiras para promover a preservação desses ecossistemas ímpares, produzindo subsídios para o gerenciamento destes ambientes.

O grande número de espécies encontradas no presente estudo (146), bem como a presença de espécies ameaçadas de extinção no Rio Grande do Sul e no Brasil (seis) e as funções ecológicas existentes, revelam o potencial da área de estudo a ser conservado. Espécies ameaçadas no Rio Grande do Sul como B. portulacoides (VU) e quase ameaçada no Brasil como $S$. crassifolius (NT) foram observadas com frequência na área de estudo, conforme parâmetros fitossociológicos (Tab. 2), indicando a importância da implementação de políticas públicas para a conservação da área, o que garantirá não apenas a preservação da biodiversidade local, mas também regional e nacional.

Os dados apresentados referentes à vegetação são de fundamental importância no processo de planejamento das ações de gestão visando à preservação deste ambiente, bem como na elaboração do Plano de Manejo do Refúgio da Vida Silvestre da Ilha dos Lobos (REVIS da Ilha dos Lobos), visto que a área de estudo integra a zona de amortecimento desta unidade de conservação.

\section{REFERÊNCIAS}

Barbier, E. B., Hacker, S. D., Kennedy, C., Koch, E. W., Stier, A. C. \& Silliman, B. R. 2011. The value of estuarine and coastal ecosystem services. Ecological monographs 81(2):169-193.

Barbieri, R. L. \& Stumpf, E. R. T. 2008. Origem e Evolução das Cultivadas. Embrapa Informação Tecnológica, Brasília. 909 p.

Barbosa, C. 2017. Invasão por Furcraea foetida (Asparagaceae) em ambientes costeiros no Brasil: uma abordagem sobre nicho ecológico, mudanças climáticas e genética de populações. Tese 111 f., Universidade Federal de Santa Catarina, Florianópolis.

BFG - The Brazilian Flora Group. 2015. Growing knowledge: an overview of Seed Plant diversity in Brazil. Rodriguesia 66: 1085-1113.

Biondo, E., Fleck, M., Kolchinski, E. M., Sant'Anna, V. \& Polesi, R. G. 2018. Diversidade e potencial de utilização de plantas alimentícias não convencionais ocorrentes no Vale do Taquari, RS. Revista Eletrônica Científica da UERGS 4(1):61-90.

Borges, A. L. \& Souza, L. S. 2004. O cultivo da bananeira. Embrapa Mandioca e Fruticultura, Cruz das Almas. 279 p.

Brack, P. 2009. Vegetação e paisagem do Litoral Norte do Rio Grande do Sul: exuberância, raridade e ameaças a biodiversidade. In Ecossistemas e biodiversidade do Litoral Norte do RS (N. L. Würdig \& S. M. F. Freitas, orgs.). Nova Prova, Porto Alegre, p. 32-55.

Brasil. 2014. Ministério do Meio Ambiente. Portaria n443, de 17 de dezembro de 2014. Lista Nacional Oficial das espécies da Flora Ameaçadas de Extinção.

Brummit, R. K. \& Powell, C. E. 1992. Authors of plant names. Kew: The Royal Botanic Gardens. 732p.

Buchmann, F. S. C., Caron, F., Lopes, R. P., Ugri, A. \& Lima, L. G. L. 2009. Panorama geológico da Planície Costeira do Rio Grande do Sul. In Quaternário do Rio Grande do Sul: integrando conhecimentos (A. M. Ribeiro, S. G. Bauermann \& C. S. Scherer, eds.). Sociedade Brasileira de Paleontologia, Porto Alegre, p. 35-56.

Calliari, L. R., Pereira, P. S., Oliveira, A. O. \& Figueiredo, S. A. 2005. Variabilidade das dunas frontais no Litoral Norte e Médio do Rio Grande do Sul, Brasil. Gravel (3):15-30.

CNCFlora- Centro Nacional de Conservação da Flora. 2017. Lista Vermelha da flora brasileira versão 2012.2. Disponível em http:// www.cncflora.jbrj.gov.br/portal/pt-br/listavermelha. Acessado em 28.10.2017.

Cordazzo, C. V. \& Costa, C. S. B. 1989. Associações vegetais das dunas frontais de Garopaba (SC). Ciência e Cultura 41(9):906-910.

Cordazzo, C. V. \& Rosa, L. S. 2010. Plantas Exóticas e Invasoras nas Dunas Costeiras do Praia do Cassino (RS), Brasil. Fepam em Revista 4(1):4-11.

Cordazzo, C. V. \& Seeliger, U. 1987. Composição e distribuição da vegetação nas dunas costeiras ao sul do Rio Grande (RS). Ciência e Cultura 39(3):321-324.

1995.Guia ilustrado da vegetação costeira do extremo sul do Brasil. FURG, Rio Grande. 2 ed. 275 p.

Costa, C. S. B., Seeliger, U. \& Cordazzo, C. V. 1991. Leaf demography and decline of Panicum racemosum populations in coastal foredunes of southern Brazil. Canadian Journal of Botany 69:1593-1599.

Duarte, C. M., Losada, I. J., Hendriks, I. E., Mazarrasa, I. \& Marbà, N. 2013. The role of coastal plant communities for climate change mitigation and adaptation. Nature Climate Change 3:961-969. 
Duarte, M. M. \& Bencke, G. A (Orgs.). 2006. Plano de Manejo do Parque Estadual de Itapeva: Relatório final. Fundação Zoobotânica, Porto Alegre. 274 p.

Falcão, M. 2000. A dinâmica espacial da cidade de Torres/RS, entre 1970 e 1998 . Boletim

Gaúcho de Geografia 26:158-172.

Fiaschi, P. \& Pirani, J. 2009. Review of plant biogeographic studies in Brazil. Journal of Systematics and Evolution 47:1-20.

Filgueiras, T. S., Brochado, A. L., Nogueira, P. E. \& Guala, G. F. 1994. Caminhamento: um método expedito para levantamentos florísticos qualitativos. Cadernos de Geociências (12):39-43.

Flora do Brasil. 2020. Flora do Brasil 2020 [em construção]. Jardim Botânico do Rio de Janeiro. Disponível em: http://floradobrasil.jbrj. gov.br/. Acessado em 28.10.2017.

Francis, J. K. 2009. Wildland shrubs of the United States and its territories: thamnic descriptions general technical report IITF-WB-1 U.S. Department of Agriculture. Forest Service International Institute of Tropical Forestry and Shrub Sciences Laboratory. Disponível em: http://www.fs.fed.us/global/iitf/wildland_shrubs.htm. Acessado em 10.10.2017.

Freitas, W. K. \& Magalhães, L. M. S. 2012. Métodos e Parâmetros para Estudo da Vegetação com Ênfase no Estrato Arbóreo. Floresta e Ambiente 19(4):520-540.

Gonzatti, F., Machado, L. \& Windisich, P. G. 2016. Distribution patterns of ferns and lycophytes in the Coastal Region of the state of Rio Grande do Sul, Brazil. Acta Botanica Brasilica 30(2):239-253.

Graciano, C. T. 2004. A Torres de concreto: da expansão turístico-urbana dos anos 70 à crise dos anos 90, um estudo sobre o processo de urbanização em Torres/RS. Dissertação 232 f., Pontifícia Universidade Católica do Rio Grande do Sul, Porto Alegre.

Hammer, Ø., Harper, D. A. T. \& Ryan, P. D. 2001. PAST - Paleontological Statistics Software Package for Education and Data Analysis, versão. 1.73. Palaeontologia Electronica 4(1):1-9.

Henriques, R. P. B., Meirelles, M. L. \& Hay, J. D. 1984. Ordenação e distribuição de espécies das comunidades vegetais na praia da restinga de Barra de Marica, Rio de Janeiro. Revista Brasileira de Botânica 7:27-36.

Hueck, K. 1953. Problemas e importância prática da fitossociologia no Estado de São Paulo. In Contribuições para a pesquisa fitossociológica paulista. Instituto de Botânica, v. 1, 20 p.

I3N Brasil. Base de dados nacional de espécies exóticas invasoras, Instituto Hórus de Desenvolvimento e Conservação Ambiental, Florianópolis - SC. Disponível em: http://i3n.institutohorus.org.br/ www. Acessado em 18.04.2019.

IBGE - Instituto Brasileiro de Geografia e Estatística. 2012. Manual Técnico da Vegetação Brasileira: Série Manuais Técnicos em Geociências. IBGE, Rio de Janeiro, 274 p.

Klein, A. S., Citadini-Zanete, V. \& Santos, R. 2007. Florística e estrutura comunitária de restinga herbácea no município de Araranguá, Santa Catarina. Biotemas 20(3):15-26.

Klein, R. M. 1984. Aspectos dinâmicos da vegetação do Sul do Brasil. Sellowia 36: 5-54.

Lacerda, L. D., Araujo, D. S. D. \& Maciel, N. C. 1993. Dry coastal ecosystems of the tropical Brazilian coast. In Dry coastal ecosystems: Africa, America, Asia and Oceania ( E. Van der Maarel, ed.). Elsevier, Amsterdam, p.477-493.

Leite, P. F. \& Klein, R. M. 1990. Vegetação. In Geografia do Brasil: Região Sul.Instituto Brasileiro de Geografia e Estatística - IBGE, Rio de Janeiro. 2 ed. p. 113-150.

Lindeman, J. C., Baptista, L. R. M., Irgang, B. E., Porto, M. L., GirardiDeiro, A. M. \& Baptista, M. L. L. 1975. Estudos botânicos no Parque Estadual de Torres, Rio Grande do Sul- Brasil. Iheringia. Série Botânica 21:15-22.

Liquete, C., Piroddi, C., Drakou, E. G., Gurney, L., Katsanevakis, S., Charef, A. \& Egoh, B. 2013. Current status and future prospects for the assessment of marine and coastas ecossyntem services: a systematic review. Plos One 8(7):1-15.

Lorenzi, H. 2013. Plantas para Jardim no Brasil: herbáceas, arbustivas e trepadeiras. Instituto Plantarum, Nova Odessa. 1120 p.

Lorenzi, H. \& Souza, V. C. 2012. Botânica sistemática: Guia Ilustrado para identificação das famílias de Fanerógamas nativas e exóticas no Brasil, baseado em APG III. Instituto Plantarum, Nova Odessa. 3 ed. 768 p.

Lorscheitter, M. L. 2003. Contribution to the Holocene history of Atlantic Rain Forest in the Rio Grande do Sul state, southern Brazil. Revista del Museo Argentino de Ciencias Naturales 5:261-27.

Martinéz, M., Intralawan, A., Vázquez, G., Pérez-Maqueo, O., Sutton, P. \& Landgrave, R. 2007. The coasts of our world: Ecological, economic and social importance. Ecological Economics 63:254-272.

Matos, D. M. S. \& Pivelo, V. R. O. 2009. Impacto das Plantas Invasoras nos Recursos Naturais de Ambientes Terrestres - Alguns casos Brasileiros. Ciência e Cultura 61(1):27-30.

Matthews, S. 2005. América do Sul invadida: a crescente ameaça das espécies exóticas invasoras. Programa Global de Espécies Invasoras. Disponível em: http://www.institutohorus.org.br/download/ gispSAmericapo.pdf. Acessado em 07.04.2019.

Mueller-Dombois, D. \& Ellenberg, H. 1974. Aims and methods of vegetation ecology. John Wiley and Sons, New York. 547 p.

Palma, C. B. \& Jarenkow, J. A. 2008. Estrutura de uma formação herbácea de dunas frontais no litoral norte do Rio Grande do Sul, Brasil. Biociências 16(2):114-124.

Parker, I. M. \& Reichard, S. H. 1997. Critical Issues in Invasion Biology for Conservation Science. In Conservation Biology for the Coming Decade (P.L. Fiedler \&. P.M. Kareiva, eds.). Chapman and Hall, New York, p. 283-305.

Pfadenhauer, J. \& Ramos, R. F. 1979. Um complexo de vegetação entre dunas e pântanos próximo a Tramandaí - Rio Grande do Sul, Brasil. Iheringia. Série Botânica 25:17-26.

Rambo, S. J. B. 1950. A porta de Torres. Anais Botânicos do Herbário Barbosa Rodrigues (2): 125- 136.

1951. A imigração da selva higrófila no Rio Grande do Sul. Anais Botânicos do Herbário Barbosa Rodrigues (3): 55-91. 1954. Historia da flora do litoral riograndense. Sellowia 6 : 113-172.

. 1956.A fisionomia do Rio Grande do Sul. Selbach, Porto Alegre. 2 ed. 456 p.

Rio Grande do Sul. 2013. Portaria da Secretaria de Meio Ambiente ${ }^{\circ} 79$, de 31 de outubro de 2013. Reconhece a Lista de espécies Invasoras do Estado do Rio Grande do Sul e demais classificações, estabelece normas de controle e dá outras providências. Diário Oficial do Estado do Rio Grande do Sul, Porto Alegre, Diário Oficial 71(212):45-48.

Rio Grande do Sul. 2014. Decreto Estadual n ${ }^{\circ} 52.109$, de $1^{\circ}$ de dezembro de 2014. Declara as espécies da flora nativa ameaçadas de extinção no Estado do Rio Grande do Sul. Diário Oficial do Estado do Rio Grande do Sul, Porto Alegre, Diário Oficial 72(233):2-11.

Rizzini, C. T. 1997. Tratado de Fitogeografia do Brasil: aspectos ecológicos, sociológicos e florísticos. Âmbito Cultural, Rio de Janeiro, 747 p.

Rosa, L. S. \& Cordazzo, C. V. 2007. Perturbações Antrópicas na Vegetação das Dunas da Praia do Cassino (RS). Furg. Cadernos de Ecologia Aquática 2(2):1-12.

Scarano, F.R. 2002. Structure, function and floristic relationships of plant communities in stressful habitats marginal to the Brazilian Atlantic rainforest. Annals of Botany 90:517-524.

Scherer, A. 2009. Estrutura e aspectos fitogeográficos de remanescentes florestais na restinga sul brasileira. Tese $130 \mathrm{f}$., Universidade Federal do Rio Grande do Sul, Porto Alegre.

Schneider, A. A. 2007. A Flora Naturalizada no Estado Rio Grande do Sul, Brasil: Herbáceas Subespontâneas. Biociências15(2):257-268.

Schneider, A. A. \& Irgang, B. E. 2005. Florística e fitossociologia de vegetação viária no município de Não-Me-Toque, Rio Grande do Sul, Brasil. Iheringia 60(1):49-62.

Schlickmann, M. B., Pereira, J. L., Vieira, G. R., Rocha, A. O. \& Martins, R. 2016. Levantamento florístico e parâmetros fitossociológicos da restinga na localidade de Morro dos Conventos, Araranguá- SC. Revista Iniciação Científica 14(1):40-51.

Seeliger, U. 1992. Coastal foredunes of southern Brazil: Physiography, Habitats, and Vegetation. In Coastal Plant Communities of Latin America (U. Seeliger, ed.). Academic Press, San Diego, p. 367-381.

Siqueira, J. C. 1992.O gênero Gomphrena L. (Amaranthaceae) no Brasil. Pesquisas Botânicas 43:90-92. 
Tomazelli, L. J. 1994. Morfologia, Organização e Evolução do Campo Eólico Costeiro do Litoral Norte do Rio Grande do Sul, Brasil. Pesquisas em Geociências 21(1):64-71.

Tomazelli, L. J., Dillenburg, S. R. \& Villwock, J. A. 2000. Late quaternary geological history of Rio Grande do Sul Coastal Plain, Southern Brazil. Revista Brasileira de Geociências 30:474-476.

Valduga, E., Gonzatti, F. \& Scur, L. 2013. A flora da restinga In Guia de identificação da flora e fauna dos ecossistemas terrestres no entorno das lagoas costeiras: municípios de Cidreira, Balneário Pinhal e Palmares do Sul (L. Scur, C. M. Joenck, orgs.). EDUCS, Caxias do Sul, p. 9-85.

Valls, J. F. M. 1975. Estudos botânicos no Parque Estadual de Torres, Rio Grande do Sul. I. Levantamento florístico da área da Guarita. Iheringia. Série Botânica 20:35-58.

Villwock, J. A. \& Tomazelli, L. J. 2007. Planície costeira do Rio Grande do Sul: gênese e paisagem atual. In Biodiversidade: Regiões da Lagoa do Casamento e dos Butiazais de Tapes, planície costeira do Rio Grande do Sul (F. G. Becker, R. A. Ramos, L. A. Moura, eds.). MMA/SBF, Brasília, p. 20-33.

Waechter, J. L. 1985.Aspectos ecológicos da vegetação da restinga no Rio Grande do Sul, Brasil. Comunicações do Museu de Ciências da PUCRS. Série Botânica 33:49-68.

1990. Comunidades vegetais das restingas do Rio Grande do Sul. In Anais do II Simpósio de Ecossistemas da Costa Sul e Sudeste Brasileira, Águas de Lindóia, 3:228-248.
1998. Epiphytic orchids in eastern subtropical South America. Proceedings of the 15th World Orchid Conference, Rio de Janeiro, Brasil. Turriers, Naturalia Publications.

2002. Padrões geográficos na flora atual do Rio Grande do Sul. Ciência e Ambiente 24:93-108.

Williamson, M. 1996. Biological Invasions. Chapman \& Hall, London, New York. 244 p.

Worm, B., Barbier, E.B., Beaumont, N., Duffy, J.E., Folke, C., Halpern, B.S., Jackson, J.B. C., Lotze, H. K., Micheli, F., Palumbi, S. R., Sala, E., Selkoe, K. A., Stachowicz, J. J.\& Watson, R. 2006. Impacts of biodiversity loss on ocean ecossystem services. Science 314:787-790.

Zardini, E. M. 1985. Revisión del género Noticastrum (CompositaeAstereae). Revista del Museo de La Plata, Tomo XIII, 86:313-424.

Zasso, L. A. 2012 Alterações na deriva litorânea e no balanço de sedimentos: molhes do rio Mampituba e praias adjacente, divisa entre os estados do Rio Grande do Sul e Santa Catarina. Dissertação 56 f., Universidade Federal do Rio Grande do Sul, Porto Alegre.

Ziller, S. R. 2001. Plantas Exóticas Invasoras: a ameaça da contaminação biológica. Ciência Hoje 30(178):77-79.

Zimmermann, T. G. 2016. Potencial de Invasão das Restingas por Casuarina equisetifolia L.: Fatores que Limitam a Regeneração da Vegetação. Tese 192 f., Instituto de Pesquisas do Jardim Botânico do Rio de Janeiro, Escola Nacional de Botânica Tropical, Rio de Janeiro.

Zuloaga, F., Guglieri, A. \& Longhi-Wagner, H. M. 2001. Panicum L. In Flora Fanerogâmica do Estado de São Paulo (M.G.L. Wanderley, G.J. Shepherd \& A.M. Giulietti, eds.). HUCITEC/ FAPESP, São Paulo 1:168-190. 